\title{
Change of Bone Mineral Density and Relationship to Clinical Parameters in Male Stroke Patients
}

\author{
Hui Dong Kim, MD, Sae Hyun Kim, MD, Dong Kyu Kim, MD, \\ Ho Joong Jeong, MD, PhD, Young Joo Sim, MD, PhD, Ghi Chan Kim, MD, PhD
}

Department of Physical Medicine and Rehabilitation, Kosin University College of Medicine, Busan, Korea

\begin{abstract}
Objective To investigate the clinical characteristics that significantly contribute to a decreased bone mineral density (BMD), the BMD changes and clinical characteristics of men who experienced a stroke between the ages of 50 years and 65 years were studied between 3 months and 4 months after the stroke.

Methods Subjects had a brain hemorrhage or a cerebral infarction. Only men aged 50 years to 65 years were included to eliminate postmenopausal osteoporosis and to eliminate the influence of senile osteoporosis. All subjects underwent a BMD test between 3 months and 4 months after their strokes. Also, patients with a medication history that might have caused a secondary osteoporosis before a stroke were excluded.

Results The BMD for the lumbar spine and hemiplegic side of the femoral neck correlated significantly with the results of the manual muscle test (MMT) of the hemiplegic lower extremity and the Modified Barthel Index (MBI) score. This result suggests that the immobility from the decreased muscle strength and the weakened daily functionality might have reduced the BMD. According to a multiple linear regression analysis, the MBI score is significantly correlated with the lumbar BMD. The BMD of the hemiplegic femoral neck is significantly correlated with the MMT and the MBI score.

Conclusion This study showed that BMD monitoring should be considered in male stroke patients, especially for patients with a high dependency in daily functions and a decreased muscle strength in the hemiplegic lower extremity.
\end{abstract}

Keywords Stroke, Bone density, Male

Department of Physical Medicine and Rehabilitation, Kosin University College of Medicine, 262 Gamcheon-ro, Seo-gu, Busan 49267, Korea. Tel: +8251-990-6261, Fax: +82-51-241-2019, E-mail: ghichan@hotmail.com

ORCID: Hui Dong Kim (http://orcid.org/0000-0002-4932-5282); Saehyun Kim (http://orcid.org/0000-0002-3151-7227); Dong Kyu Kim (http://orcid. org/0000-0002-9484-6562); Ho Joong Jeong (http://orcid.org/0000-0002-0607-2799); Young-Joo Sim (http://orcid.org/0000-0002-0640-8766); Ghi Chan Kim (http://orcid.org/0000-0002-4954-3175).

(c) This is an open-access article distributed under the terms of the Creative Commons Attribution Non-Commercial License (http://creativecommons.org/ licenses/by-nc/4.0) which permits unrestricted noncommercial use, distribution, and reproduction in any medium, provided the original work is properly cited. Copyright $\odot 2016$ by Korean Academy of Rehabilitation Medicine 


\section{INTRODUCTION}

Osteoporosis is a skeletal disorder characterized by a compromised bone strength and an increased risk of fracture [1]. Primary osteoporosis occurs in people with no endocrinopathy or other diseases that would account for the change of bone mass. Postmenopausal osteoporosis results from increases of bone resorption and the blood-calcium levels, and decreased parathyroidhormone levels and intestinal calcium absorption after menopause. Senile osteoporosis is the physiological bone loss from aging. Secondary osteoporosis, which includes endocrinal, nutritional, hereditary, and focal osteoporosis, is the result of certain medical conditions, diseases, or medications that interfere with the attainment of the peak bone mass, or that may predispose a person to accelerated bone loss [2]. In 1994, the World Health Organization proposed guidelines for the diagnosis of osteoporosis based on bone mineral density (BMD) measurements. A decrease of the bone mass within -2.5 and -1.0 on the standard deviation of the normal average bone mass is defined as osteopenia, and a decrease lower than -2.5 is osteoporosis. Osteoporosis that is accompanied by a fracture is considered severe osteoporosis [3].

Osteoporosis and the accompanying hip-joint or wrist fractures are severe complications for stroke patients, whose risk of a hip-joint fracture is two times to four times higher than that of normal adults, and who are associated with an incidence rate of $8 \%$ to $29 \%$ [4]. Even when complications are prevented, osteoporosis is usually diagnosed in stroke patients after the occurrence of a fracture, and this can lead to a gait disturbance and a substantial decline of the patient's functional status [5]. The mechanism of the decreased bone mass on the paretic side of hemiplegic stroke patients is related to a variety of factors [6]. The motor deficiency and the level of functional dependency during the hospital stay affect the bone-mass decrease, whereby a statistically significant correlation has been identified between the serum calcium and the Barthel Index. The bone-mass decrease on the paretic side seems to be caused by an increase of the bone resorption during the immobilization $[7,8]$. Insufficient nutrient intake, a vitamin D deficiency from the lack of exposure to sunlight, and compensative hyperparathyroidism also increase the bone resorption and decrease the BMD $[9,10]$.
During the first month after a stroke, the bone demineralization on the paralyzed side does not significantly change, but it reaches a maximum within a 3- to 4-month period $[11,12]$. The demineralization continues slowly for up to 1 year $[7,13]$, and it remains steady after 1 year [8]. The subsequent long-term effect of a stroke on bone demineralization remains unclear [11]. Nonetheless, in stroke patients, the degree of bone loss depends on the length of the paralysis [14].

Although many studies have identified the causes of bone demineralization in stroke patients, the participants are mainly both men and women, the average period of illness for the men is as long as 4 years [12], or the studies were conducted on the patients in the acute phase within 1 month of the stroke, or in the chronic phase of over 1 year after the stroke [15]. Previous studies confirmed that the postmenopausal period is the strongest factor affecting the decrease of the BMD $[16,17]$, and BMD is affected by several different treatments $[18,19]$; therefore, the present study comprises only male stroke patients to investigate the correlation between the stroke and BMD. The men were studied 3 to 4 months after their strokes, during which time they showed significant demineralization activity. Men who were within 1 month of a stroke were excluded because significant BMD changes are not shown in that period [15].

The authors hypothesized that if the BMD changes and clinical characteristics of the men who had a stroke between the ages of 50 years and 65 years were studied 3 months to 4 months after their strokes, the effects of postmenopausal osteoporosis and senile osteoporosis would be avoided. The authors further hypothesized that the factors that are closely involved in the decreasing of the BMD would be observed with this study design. To investigate these hypotheses and determine the factors that influence a decrease of the BMD in patients after the stroke, the present study examined the correlations between a decreased BMD and the clinical parameters of the stroke.

\section{MATERIALS AND METHODS}

\section{Subjects}

The study subjects had experienced a cerebral hemorrhage or infarction and were admitted to rehabilitation centers for treatment at Kosin University Gospel Hospital 
from January 2010 to January 2014. The first stroke was verified through computed tomography (CT) and magnetic resonance imaging (MRI). Men aged from 50 years to 65 years were included to eliminate postmenopausal osteoporosis and to eliminate the influence of senile osteoporosis, and they underwent BMD measurements 3 months to 4 months after their stroke. The control group included men from the university healthcare centers who were in the same age group with no history of stroke, no history of drug intake that would affect osteoporosis, and no medical history indicating osteoporosis. The BMD in the experimental group was measured using dual-energy X-ray absorptiometry (DXA) (Lunar DPXL/PED; Lunar Radiation, Madison, WI, USA) at L1-L4 and the femoral neck of the hemiplegic lower limb. The BMD-test results were analyzed using the T-score, absolute BMD $\left(\mathrm{g} / \mathrm{cm}^{2}\right)$, and DXA-test results from the follow-up.

As previously stated, patients younger than 50 years or older than 65 years and patients who had a stroke less than 3 months previously were excluded. The authors also excluded 12 patients with a medication history that might have caused a secondary osteoporosis before the stroke; moreover, patients with a calcium, vitamin D, and/or bisphosphonate-related medication history and patients with a previous history of stroke were excluded.

\section{Methods}

The Institutional Review Board approval was granted by Kosin University College of Medicine prior to the commencement of the study activities. This retrospective study collected the age, height, and body mass index (BMI) of each of the patients. The muscle strength of the hemiplegic lower extremity (hip extensor and knee extensor) was evaluated using a manual muscle test (MMT). Two groups were formed as follows: One group showed MMT grades for both the hip extensor and the knee extensor that are above the F grade, and the other group showed an MMT grade of either the hip extensor or the knee extensor that is below the F grade. The degree of spasticity in the hemiplegic lower extremity (hip flexor or hip extensor) was evaluated using the Modified Ashworth Scale (MAS); here, the higher one between the hip flexor and the hip extensor was analyzed. A loss of proprioception regarding the joint position was tested through passive vertical-motion exercises of the fingers and toes on both sides. The daily-functionality status was evaluated using the Modified Barthel Index (MBI), and the cognitive function was evaluated using the Korean version of the Mini-Mental State Examination (MMSE-K). The possible use of the medications commonly used by stroke patients and that may cause osteoporosis was investigated, specifically steroids, anticoagulants, antiplatelets (warfarin, heparin), and anticonvulsants (phenytoin, phenobarbital, primidone, carbamazepine, and valproic acid); stroke-related medicines were only considered if they were continuously used for more than 1 month. The mobility status was classified according to independent walking, independent walking under supervision, walking with assistance, immobility, and wheelchair use.

The participants were classified according to their BMI, which is calculated as the weight divided by the square of the height. A BMI below $18.5 \mathrm{~kg} / \mathrm{m}^{2}$ is underweight, 18.5 to $22.9 \mathrm{~kg} / \mathrm{m}^{2}$ is normal, 23 to $24.9 \mathrm{~kg} / \mathrm{m}^{2}$ is overweight, and more than $25 \mathrm{~kg} / \mathrm{m}^{2}$ is obese. By varying the resistance a muscle withstood, the MMT was used by the authors to classify the degree of muscle strength, and a grade of 0 to 5 was determined using the factors of gravity, resistance, and range of motion. The MAS is the most commonly used method for evaluating the degree of spasticity, and it was also classified from 0 to 5 . The MMSE- $K$, a revised version of the MMSE that was developed in 1975 in the United States, was used to evaluate the cognitive function and short-term dementia.

\section{Statistical analysis}

The Mann-Whitney U-test was used to compare the Tscores and the absolute BMD data between the control and experimental groups. The Spearman correlation analysis was used to determine the presence of any correlations in the experimental group for the BMI, physical characteristics, physical examination, and clinical parameters. Linear regression was used to determine the independent influence of each risk factor on the BMD; here, $p$-value is statistically significant below 0.05 . All of the data were analyzed using the SPSS for Windows ver. 14.0 (SPSS Inc., Chicago, IL, USA).

\section{RESULTS}

\section{General characteristics of the participants}

The general characteristics of the experimental group are presented in Table 1. The group comprises 21 men 
Table 1. Baseline demographics and clinical parameters of male patients with stroke

\begin{tabular}{|cc|}
\hline \multicolumn{1}{|c|}{ Characteristic } & Value \\
\hline Age $(\mathrm{yr})$ & $57.8 \pm 8.3$ \\
\hline BMI $\left(\mathrm{kg} / \mathrm{m}^{2}\right)$ & $23.6 \pm 2.4$ \\
\hline Stroke duration (day) & $104.6 \pm 13.8$ \\
\hline MMT of hip and knee extensor & 13 \\
\hline F grade and over & 8 \\
\hline Below F grade & $0.8 \pm 1.2$ \\
\hline MAS & $18.5 \pm 7.6$ \\
\hline MMSE-K & $51.7 \pm 23.8$ \\
\hline MBI & 7 \\
\hline Drug use & 14 \\
\hline No & \\
\hline Yes & 15 \\
\hline Loss of proprioception & 6 \\
\hline No & \\
\hline Yes & 5 \\
\hline Ambulation & 9 \\
\hline Independent or supervision & 7 \\
\hline Dependent & \\
\hline Wheelchair use & \\
\hline
\end{tabular}

Values are presented as mean \pm standard deviation or number.

BMI, body mass index; MMT, manual muscle test; MAS, Modified Ashworth Scale; MMSE-K, Korean version of Mini-Mental Status Examination; MBI, Modified Barthel Index.

with the average age of $57.8 \pm 8.3$ years, and the average BMI is $23.6 \pm 2.4 \mathrm{~kg} / \mathrm{m}^{2}$. The MMT for the hip and knee joints, which are important for standing and walking, identified 13 patients with both a hip extensor and a knee extensor the $\mathrm{F}$ grade and over, and eight patients with a hip extensor or a knee extensor below the F grade. The average spasticity is $0.8 \pm 1.2$ according to the MAS at the hip flexor or extensor, and the average cognitive function is $18.5 \pm 7.6$ according to the MMSE-K. The evaluation of the daily-functionality status using the MBI showed an average of $51.7 \pm 23.8$, which requires moderate assistance for daily tasks. For proprioception, which is important for standing and walking, 6 patients indicated a loss of a sense of position and 15 did not. Stroke-related medications were taken for over a month by 14 participants; whereas 7 had not taken medications. Five patients could walk independently or independently under supervision,
9 walked with minimum-to-maximum assistance, and 7 required a wheelchair.

Bone density difference between control and experimental groups

Men between the ages of 50 years and 65 years with no history of stroke who underwent the BMD test at the university healthcare centers formed the control group. The stroke patients included in this study underwent the BMD test at the time of transfer or after their admission to the Department of Rehabilitation Medicine, and the average time period that had elapsed after the stroke is $\mathbf{1 0 4 . 6}$ days. The lowest values for the lumbar and hemiplegic side of the femoral neck were measured; the T-scores and absolute BMD $\left(\mathrm{g} / \mathrm{cm}^{2}\right)$ values of the lumbar of the femoral neck for the comparison group are $-0.48 \pm 1.45$, $-0.29 \pm 1.02,1.12 \pm 0.18$, and $0.94 \pm 0.12$, respectively, and for the stroke patients, they are $-1.71 \pm 1.29,-1.04 \pm 1.18$, $0.95 \pm 0.16$, and $0.82 \pm 0.14$, respectively. The difference between the bone-density values of the two groups is significant.

Correlation between bone density and clinical parameters in stroke patients

The clinical parameters and the BMD results of the groups were analyzed using the Spearman correlation. The BMD results of the stroke group for the lumbar and hemiplegic side of the femoral neck correlated significantly with the MMT results of the hemiplegic lower extremity and the MBI results, suggesting that the immobility from a decreased muscle strength and a weakened daily functionality from the stroke reduced the BMD. No significant correlations were observed for the other clinical parameters (Tables 2, 3).

\section{Multiple linear regression analysis}

For the multiple linear regression analysis, the dependent variables for the stroke patients are the T-score and the absolute BMD, and the independent variables are the BMD, MMT, MAS, loss of proprioception, MBI, MMSE$\mathrm{K}$, walking status, and BMI. The MBI is an independent variable that is significantly correlated with the lumbar BMD in the stroke patients. In these patients, the BMD of the hemiplegic femoral neck is significantly correlated with the MMT and the MBI. No other independent variables showed significant correlations (Tables 4, 5). 
Table 2. Correlation between T-scores and clinical parameters in male patients with stroke

\begin{tabular}{|c|c|c|c|c|}
\hline & \multicolumn{2}{|c|}{ L-spine } & \multicolumn{2}{|c|}{ Femur neck } \\
\hline & $\mathbf{r}$ & p-value & $\mathbf{r}$ & p-value \\
\hline $\begin{array}{l}\text { MMT of } \\
\text { hip and knee }\end{array}$ & 0.503 & $0.002^{* *}$ & 0.517 & $0.019^{*}$ \\
\hline MAS & -0.072 & 0.770 & -0.324 & 0.176 \\
\hline $\begin{array}{l}\text { Loss of } \\
\text { proprioception }\end{array}$ & -0.228 & 0.363 & -0.193 & 0.442 \\
\hline MBI & 0.657 & $0.024^{*}$ & 0.543 & $0.013^{*}$ \\
\hline MMSE-K & 0.122 & 0.642 & 0.256 & 0.320 \\
\hline Drug use & 0.055 & 0.819 & -0.191 & 0.420 \\
\hline Ambulation & 0.111 & 0.641 & 0.117 & 0.624 \\
\hline BMI & 0.262 & 0.264 & 0.254 & 0.281 \\
\hline
\end{tabular}

MMT, manual muscle test; MAS, Modified Ashworth Scale; MMSE-K, Korean version of Mini-Mental Status Examination; MBI, Modified Barthel Index; BMI, body mass index.

${ }^{*} \mathrm{p}<0.05,{ }^{* *} \mathrm{p}<0.01$.

Table 3. Correlation between absolute BMD $\left(\mathrm{g} / \mathrm{cm}^{2}\right)$ and clinical parameters in male patients with stroke

\begin{tabular}{lccccc}
\hline & \multicolumn{2}{c}{ L-spine } & & \multicolumn{2}{c}{ Femur neck } \\
\cline { 2 - 3 } \cline { 5 - 6 } & $\mathbf{r}$ & p-value & & r & p-value \\
\hline $\begin{array}{l}\text { MMT of } \\
\quad \text { hip and knee }\end{array}$ & 0.555 & $0.011^{*}$ & & 0.568 & $0.009^{* *}$ \\
MAS & -0.059 & 0.810 & & -0.302 & 0.209 \\
$\begin{array}{l}\text { Loss of } \\
\text { proprioception }\end{array}$ & -0.114 & 0.654 & & -0.204 & 0.416 \\
MBI & 0.688 & $0.001^{* *}$ & 0.591 & $0.006^{* *}$ \\
MMSE-K & -0.139 & 0.595 & & -0.300 & 0.242 \\
Drug use & -0.173 & 0.467 & & -0.245 & 0.297 \\
Ambulation & 0.144 & 0.544 & 0.075 & 0.753 \\
BMI & 0.370 & 0.108 & 0.355 & 0.125 \\
\hline
\end{tabular}

MMT, manual muscle test; MAS, Modified Ashworth Scale; MMSE-K, Korean version of Mini-Mental Status Examination; MBI, Modified Barthel Index; BMI, body mass index; BMD, bone mineral density.

${ }^{*} \mathrm{p}<0.05,{ }^{* *} \mathrm{p}<0.01$.

\section{DISCUSSION}

Osteoporosis is a musculoskeletal disease that increases the fracture risk because of the weakening of the BMD, and it has a clinically significant meaning [1]. An important goal of osteoporosis treatment is the prevention of fractures, which are caused by falls and a decreased BMD
Table 4. Multiple regression analysis for T-score and clinical parameters in male patients with stroke

\begin{tabular}{|c|c|c|c|c|}
\hline & \multicolumn{2}{|c|}{ L-spine } & \multicolumn{2}{|c|}{ Femur neck } \\
\hline & $\beta$ & p-value & $\beta$ & p-value \\
\hline $\begin{array}{l}\text { MMT of hip } \\
\text { and knee }\end{array}$ & 0.543 & 0.078 & 0.583 & $0.016^{*}$ \\
\hline MBI & 0.489 & $0.021^{*}$ & 0.598 & $0.014^{*}$ \\
\hline Adjusted $\mathrm{R}^{2}$ & \multicolumn{2}{|c|}{0.591} & \multicolumn{2}{|c|}{0.476} \\
\hline
\end{tabular}

$\beta$, standardized regression coefficient; MMT, manual muscle test; MBI, Modified Barthel Index.

${ }^{*} \mathrm{p}<0.05$.

Table 5. Multiple regression analysis for absolute BMD $\left(\mathrm{g} / \mathrm{cm}^{2}\right)$ and clinical parameters in male patients with stroke

\begin{tabular}{|c|c|c|c|c|}
\hline & \multicolumn{2}{|c|}{ L-spine } & \multicolumn{2}{|c|}{ Femur neck } \\
\hline & $\beta$ & p-value & $\beta$ & p-value \\
\hline $\begin{array}{l}\text { MMT of hip } \\
\text { and knee }\end{array}$ & 0.176 & 0.474 & 0.557 & $0.046^{*}$ \\
\hline MBI & 0.609 & $0.024^{*}$ & 0.571 & $0.040^{*}$ \\
\hline Adjusted $\mathrm{R}^{2}$ & \multicolumn{2}{|c|}{0.688} & \multicolumn{2}{|c|}{0.704} \\
\hline
\end{tabular}

$\beta$, standardized regression coefficient; MMT, manual muscle test; MBI, Modified Barthel Index; BMD, bone mineral density.

${ }^{*} \mathrm{p}<0.05$.

in osteoporosis patients. In stroke patients, a decreased BMD from an increased bone resorption occurs as the muscle tone decreases and during immobilization [7]. These factors increase the risk of falls because of a loss of balance and a decreased muscle strength, exposing people who have had a stroke to a higher fracture risk. With the joint contracture and nutritional problems that occur later, the incidence of osteoporosis increases [20]. According to the Korea National Health and Nutrition Examination Survey IV \& V, the osteoporosis prevalence in the general Korean population that is over the age of 50 years and who are not in a hospital is $7.3 \%$ for men and $38.0 \%$ for women [21]; however, a previous study by Kim et al. [22] on stroke patients in Korea reported an osteoporosis prevalence of $43.8 \%$ in 48 people, 1 month after the stroke. Kwon et al. [23] reported osteoporosis prevalence of $78.1 \%$ in female stroke patients and $22.9 \%$ in male stroke patients from 121 patients who were over the age of 56 years; this is above the average prevalence of the general population. 
Although postmenopausal osteoporosis was eliminated in the present study through the inclusion of only male stroke patients, and senile osteoporosis was eliminated through the inclusion of only patients between the ages of 50 years and 65 years, the osteoporosis prevalence is still $\mathbf{3 8 . 1 \%}$, which is significantly higher than that of the normal comparison group (16.5\%). A significant BMD decrease was found among the stroke patients when compared with the levels of the control group. These results indicate that certain stroke-related factors led to a decreased BMD between 3 months and 4 months after the stroke.

In the linear regression analysis, the BMD on the hemiplegic side of the femoral neck among the stroke patients is independently related to the MMT of the hemiplegic lower extremity and the daily-functionality status of the men 3 months to 4 months after the stroke. The lumbar BMD is also significantly correlated with the daily-functionality status. These results correspond to previous studies. Yavuzer et al. [6] studied the relationships between the BMD and the clinical parameters of motor deficiency and dependency in daily living among 32 hemiplegic stroke patients with an average age of 62.4 years during their hospital admissions, and these factors were associated with bone-mineral loss. Sato et al. [7] observed a correlation between the bone-resorption markers and the MBI, degree of hemiplegia, and serumcalcium level, and reported a decrease of the BMD in the hemiplegic limbs from an increased bone resorption that occurs during immobilization. Other studies reported a possible decrease of the BMD that is caused by the hemiplegia and temporary immobilization in patients with a spinal cord injury or who have experienced a stroke [24].

Greater decreases of the BMD were observed in the stroke patients of this study whose muscle strength in the hemiplegic lower extremity could not overcome gravity. This result confirmed that the muscle strength from hemiplegia is directly related to the BMD in stroke patients, and that it is an important risk factor in this period. There is no statistical significance between the classification of ambulation and the decrease of the BMD, as the authors thought that an ambulation dependence in the early phase after the stroke does not sufficiently represent a functional ambulation in terms of the activities of daily living. The correlation between the BMD and the MBI proved that the more that the stroke patient relied on others for daily functions, the greater the bone loss that occurred in the beginning.

The muscle strength of the hemiplegic limb did not show a significant correlation with the lumbar BMD in the linear regression analysis, but it did show a significant correlation with the MBI. Although a decline of the muscle strength of the hemiplegic limb is generally a major cause of a BMD decline, in the present study, the initial bone density after an acute stroke was also affected by a number of factors that include the MBI. This finding implies that the physical incompetence that is caused by essential absolute bed rest, and the ensuing decline of the daily-functionality status, is closely related to bone loss in early phase stroke patients.

It is assumed that the use of stroke-related medicine is a risk factor for a BMD decrease [25,26]; however, in this study, the group with the continuous drug usage over 1 month did not show significant differences compared with the non-drug-usage group. The occurrence of this result is probably because the medication period for the drug-use group is only 3 months to 4 months and the doses are not high. The degree of spasticity, loss of a sense of position, and walking functionality are not significantly related to a decreased bone density.

The previous studies found no significant decrease of the bone density in men who had experienced a stroke and were studied within 1 month of the stroke [15]. BMD decreases reportedly accelerate at an average of 3 months to 4 months after the stroke $[11,12]$. Hamdy et al. [27] reported that the BMD in the hemiplegic limb decreased by $4.6 \%$ at 11.3 weeks after the stroke. Therefore, the authors did not study acute-stroke patients at around 1 month, but instead studied the correlation between bone density and the clinical parameters for stroke patients 3 months to 4 months after the stroke. Compared with the previous studies, the BMD changes for the acute-stroke patients of the present study are not large; however, the dependency on others is sustained for more than three months, and low muscle strength is an important factor in terms of a decreased bone density.

Although a longitudinal survey may be an excellent method for finding the relationships between a decrease of the bone density and clinical characteristics for stroke patients over time, this retrospective study was conducted to discover the direct clinical characteristics of the stroke that are related to a BMD decrease, whereby post- 
menopausal and senile strokes are eliminated. Although the BMD might not decrease during the acute phase within 1 month after the stroke, a decrease of the daily functionality that is sustained for 3 to 4 months, as seen in this study, contributes to a decreased bone density. In the initial brain rehabilitation, a continuous performance of the BMD test and the proper management of early stage stroke patients are important, especially for those patients with a low daily-functionality status.

After the onset of the stroke, the patients experience a decreased BMD and were diagnosed with osteoporosis during different periods of the rehabilitation stage. This study excluded the potential effects of postmenopausal and senile osteoporosis, and a more direct correlation between the stroke and osteoporosis was observed in the men. The linear regression analysis of the stroke group showed that a decreased MMT of the hemiplegic lower extremity and a decreased daily functionality are independently correlated with a decreased BMD. BMD monitoring should therefore be conducted continuously on stroke patients during the early stages, and especially for patients with a high dependency regarding daily functions and a low muscle strength in the hemiplegic lower extremity. Proper management is important during the initial stroke rehabilitation to help with osteoporosis, and for the prevention and treatment of the fractures that are caused by this condition.

\section{CONFLICT OF INTEREST}

No potential conflict of interest relevant to this article was reported.

\section{REFERENCES}

1. NIH Consensus Development Panel on Osteoporosis Prevention, Diagnosis, and Therapy. Osteoporosis prevention, diagnosis, and therapy. JAMA 2001; 285:785-95.

2. Lane JM, Riley EH, Wirganowicz PZ. Osteoporosis: diagnosis and treatment. Instr Course Lect 1997;46:44558.

3. Kanis JA, Gluer CC. An update on the diagnosis and assessment of osteoporosis with densitometry. Committee of Scientific Advisors, International Osteoporosis Foundation. Osteoporos Int 2000;11:192-202.
4. Paker N, Bugdayci D, Tekdos D, Dere C, Kaya B. Relationship between bone turnover and bone density at the proximal femur in stroke patients. J Stroke Cerebrovasc Dis 2009;18:139-43.

5. Iwamoto J, Tsukimura T, Takeda T. Bone mineral density of metatarsus in hemiplegic subjects. Am J Phys Med Rehabil 1999;78:202-7.

6. Yavuzer G, Ataman S, Suldur N, Atay M. Bone mineral density in patients with stroke. Int J Rehabil Res 2002;25:235-9.

7. Sato Y, Kuno H, Kaji M, Ohshima Y, Asoh T, Oizumi K. Increased bone resorption during the first year after stroke. Stroke 1998;29:1373-7.

8. Sato Y, Fujimatsu Y, Kikuyama M, Kaji M, Oizumic K. Influence of immobilization on bone mass and bone metabolism in hemiplegic elderly patients with a long-standing stroke. J Neurol Sci 1998;156:205-10.

9. Ko MH, Lee RJ, Lim ST, Park SH, Seo JH. Characteristics of bone mineral density in hemiplegic upper extremity. J Korean Acad Rehabil Med 2004;28:214-9.

10. Poplingher AR, Pillar T. Hip fracture in stroke patients: epidemiology and rehabilitation. Acta Orthop Scand 1985;56:226-7.

11. Hamdy RC, Moore SW, Cancellaro VA, Harvill LM. Long-term effects of strokes on bone mass. Am J Phys Med Rehabil 1995;74:351-6.

12. Takamoto S, Masuyama T, Nakajima M, Seikiya K, Kosaka $\mathrm{H}$, Morimoto $\mathrm{S}$, et al. Alterations of bone mineral density of the femurs in hemiplegia. Calcif Tissue Int 1995;56:259-62.

13. Ramnemark A, Nyberg L, Lorentzon R, Englund U, Gustafson Y. Progressive hemiosteoporosis on the paretic side and increased bone mineral density in the nonparetic arm the first year after severe stroke. Osteoporos Int 1999;9:269-75.

14. del Puente A, Pappone N, Mandes MG, Mantova D, Scarpa R, Oriente P. Determinants of bone mineral density in immobilization: a study on hemiplegic patients. Osteoporos Int 1996;6:50-4.

15. Sahin L, Ozoran K, Gunduz OH, Ucan H, Yucel M. Bone mineral density in patients with stroke. Am J Phys Med Rehabil 2001;80:592-6.

16. Kim GC, Jeong HJ, Roh KH, Chung HC, Kim HY, Lee HN. The changes of bone mineral density, biochemical bone markers, and lipid profiles according to duration of menopause and HRT. J Korean Acad Rehabil 
Med 2001;25:491-7.

17. Kim GC, Jeong HJ, Jeong SW, Chung HC. Relationship among estradiol, lipid profile, biochemical markers, and bone mineral density according to postmenopausal period. J Korean Acad Rehabil Med 2000;24:318-25.

18. Kim GC, Jeong HJ, Ha HS, Lee SJ. Therapeutic effect according to estrogen dosage on combined therapy with estrogen and alendronate in postmenopausal osteoporosis. J Korean Acad Rehabil Med 2006;30:247-53.

19. Chung SM, Kim GC. Effects of the combined therapy with raloxifene and low-dose intermittent fluoride for two years in postmenopausal women with osteoporosis. J Korean Acad Rehabil Med 2007;31:207-12.

20. Sato Y. Abnormal bone and calcium metabolism in patients after stroke. Arch Phys Med Rehabil 2000;81:117-21.

21. Park EJ, Joo IW, Jang MJ, Kim YT, Oh K, Oh HJ. Prevalence of osteoporosis in the Korean population based on Korea National Health and Nutrition Examina- tion Survey (KNHANES), 2008-2011. Yonsei Med J 2014;55:1049-57.

22. Kim HW, Kang E, Im S, Ko YJ, Im SA, Lee JI. Prevalence of pre-stroke low bone mineral density and vertebral fracture in first stroke patients. Bone 2008;43: 183-6.

23. Kwon J, Lim SH, Kim HW, Ko YJ, Ryu JE, Chung YC, et al. The pre-stroke bone mineral density in first stroke patients. J Korean Acad Rehabil Med 2010;34:285-9.

24. Kim G. Immobilization osteoporosis. Osteoporosis 2011;9:1-17.

25. Panday K, Gona A, Humphrey MB. Medication-induced osteoporosis: screening and treatment strategies. Ther Adv Musculoskelet Dis 2014;6:185-202.

26. Mazziotti G, Canalis E, Giustina A. Drug-induced osteoporosis: mechanisms and clinical implications. Am J Med 2010;123:877-84.

27. Hamdy RC, Krishnaswamy G, Cancellaro V, Whalen K, Harvill L. Changes in bone mineral content and density after stroke. Am J Phys Med Rehabil 1993;72:188-91. 